\title{
Erratum to: Prediction of outcome in isolated methylmalonic acidurias: combined use of clinical and biochemical parameters
}

\author{
F. Hörster • S. F. Garbade • T. Zwickler • H. I. Aydin • O. A. Bodamer • \\ A. B. Burlina • A. M. Das • J. B. C. De Klerk • C. Dionisi-Vici • S. Geb • \\ G. Gökcay • N. Guffon - E. M. Maier • E. Morava - J. H. Walter • B. Schwahn • \\ F. A. Wijburg • M. Lindner • S. Grünewald • M. R. Baumgartner • S. Kölker
}

Published online: 31 October 2009

(C) SSIEM and Springer 2009

Erratum to: J Inherit Metab Dis 2009

DOI 10.1007/s10545-009-1189-6

Although not mentioned in the article, the authors would like to note that Drs Baumgartner and Kölker have contributed equally to this study and thus have agreed on shared senior authorship.

The online version of the original article can be found at http:/ dx.doi.org/10.1007/s10545-009-1189-6

F. Hörster $(\bowtie) \cdot$ S. F. Garbade · T. Zwickler · M. Lindner •

S. Kölker

Department of General Pediatrics,

Division of Inborn Metabolic Diseases,

University Children's Hospital Heidelberg,

Im Neuenheimer Feld 430,

D-69120 Heidelberg, Germany

e-mail: friederike.hoerster@med.uni-heidelberg.de

H. I. Aydin

Department of Metabolism, Children's Hospital,

Hacettepe University Ankara,

Ankara, Turkey
O. A. Bodamer

Department of Paediatrics, Allgemeines Krankenhaus,

Vienna, Austria

A. B. Burlina

Department of Paediatrics, Division of Metabolic Disorders, University Hospital Padova,

Padova, Italy

A. M. Das

Department of Paediatrics II,

Medizinische Hochschule Hannover,

Hannover, Germany 
J. B. C. De Klerk

Sophia Children's Hospital, Erasmus Medical Centre, Rotterdam, The Netherlands

C. Dionisi-Vici

Division of Metabolism, Bambino Gesù Children's Hospital, Rome, Italy

\section{S. Geb}

University Children's Hospital I,

Frankfurt, Germany

\section{G. Gökcay}

Department of Nutrition and Metabolism,

Istanbul University Medical Faculty Children's Hospital,

Istanbul, Turkey

\section{N. Guffon}

Centre de Référence des Maladies Héréditaires du Métabolisme, Hôpital Femme Mère Enfant,

Lyon, France

\section{E. M. Maier}

Dr. von Hauner Children's Hospital,

Munich, Germany
E. Morava

Radboud University Medical Centre Nijmegen,

Nijmegen, The Netherlands

J. H. Walter

Willink Unit, Royal Manchester Children's Hospital, Manchester, UK

B. Schwahn

Department of General Pediatrics,

University Children's Hospital,

Düsseldorf, Germany

\section{F. A. Wijburg}

Department of Pediatrics, Academic Medical Centre, University Hospital,

Amsterdam, The Netherlands

S. Grünewald

Metabolic Unit, Great Ormond Street Hospital,

London, UK

M. R. Baumgartner

Metabolism and Molecular Paediatrics,

University Children's Hospital,

Zurich, Switzerland 